\title{
Galactic mass-losing AGB stars probed with the IRTS. II
}

\author{
T. Le Bertre ${ }^{1}$, M. Tanaka ${ }^{2,3}$, I. Yamamura ${ }^{2}$, and H. Murakami ${ }^{2}$ \\ 1 LERMA, UMR 8112, Observatoire de Paris, 61 Av. de l'Observatoire, 75014 Paris, France \\ 2 Institute of Space and Astronautical Science, 3-1-1 Yoshinodai, Sagamihara, Kanagawa 229-8510, Japan \\ ${ }^{3}$ National Astronomical Observatory, Mitaka, Tokyo, 181-8588, Japan
}

Received 21 January 2003/ Accepted 11 March 2003

\begin{abstract}
We are using the 2002 data-release from the Japanese space experiment IRTS to investigate the spatial distribution of galactic mass-losing $\left(>2 \times 10^{-8} M_{\odot} \mathrm{yr}^{-1}\right)$ AGB stars and the relative contribution of C-rich and O-rich ones to the replenishment of the ISM. Our sample contains $126 \mathrm{C}$-rich and 563 O-rich sources which are sorted on the basis of the molecular bands observed in the range 1.4-4.0 $\mu \mathrm{m}$, and for which we estimate distances and mass loss rates from near-infrared photometry $(K$ and $L^{\prime}$ ).

There is a clear dependence on galactocentric distance, with O-rich sources outnumbering C-rich ones for $r_{\mathrm{GC}}<8 \mathrm{kpc}$, and the reverse for $r_{\mathrm{GC}}>10 \mathrm{kpc}$. The contribution to the replenishment of the ISM by O-rich AGB stars relative to C-rich ones follows the same trend. Although they are rare ( 10\% in our sample), sources with $10^{-6} M_{\odot} \mathrm{yr}^{-1}<\dot{M}<10^{-5} M_{\odot} \mathrm{yr}^{-1} \mathrm{dominate}$ the replenishment of the ISM by contributing to $\sim 50 \%$ of the total of the complete sample.

We find 2 carbon stars at more than $1 \mathrm{kpc}$ from the Galactic Plane, that probably belong to the halo of our Galaxy.
\end{abstract}

Key words. stars: carbon - stars: mass-loss - stars: AGB and post-AGB - ISM: evolution - Galaxy: solar neighbourhood infrared: stars

\section{Introduction}

Red giants lose matter at a high rate during their evolution on the AGB (Asymptotic Giant Branch). This phenomenon is important for the evolution of the Interstellar Medium (ISM), which is replenished by the mass loss from all kinds of stars, as well as by the galactic infall, and whose composition is evolving depending on the nature of the supplied material. As the atmospheres of AGB stars are enriched by convection of elements processed in their interiors, the contribution of AGB stars to some species in the ISM, e.g. s-elements and carbon, can be very important. Also their winds are dusty and they are believed to be the main source of the injection of dust in the ISM.

We are exploiting the data obtained with the Japanese experiment IRTS (Infrared Telescope in Space) to evaluate the contribution of galactic mass-losing AGB stars to the cosmic cycle of matter. In our first paper (Le Bertre et al. 2001, Paper I) we used a sample of 126 AGB sources extracted from a preliminary data-release. On the basis of this sample, a dependence of the relative contribution of oxygen-rich sources to carbon-rich

Send offprint requests to: T. Le Bertre,

e-mail: Thibaut.LeBertre@obspm.fr

* The complete Tables 3 and 4 are available in electronic form at the CDS via anonymous ftp to

cdsarc.u-strasbg.fr $(130.79 .128 .5)$ or via

http://cdsweb.u-strasbg.fr/cgi-bin/qcat?J/A+A/403/943 ones on the galactocentric distance has been reported. Also in this sample, most of the replenishment of the ISM is the effect of stars with large mass loss rates $\left(>10^{-6} M_{\odot} \mathrm{yr}^{-1}\right)$. In this selection only 2 sources contributed one fourth of the total sample mass loss rate, demonstrating that the analysis was suffering from small number effects. Here we extend our study using a larger sample in order to refine our preliminary analysis. We first describe the method that we have used to extract the data and then present our new results.

\section{Presentation of the data}

\subsection{The IRTS mission and the NIRS}

The IRTS is a $15-\mathrm{cm}$ diameter helium-cooled telescope. It operated from space in 1995 during 26 days and surveyed $~ 7 \%$ of the sky (2700 $\mathrm{deg}^{2}$, Fig. 1) with 4 infrared instruments (Murakami et al. 1996). For our purpose we use the data acquired by the Near-Infrared Spectrometer (NIRS). The NIRS is a low-resolution grating spectrometer that covers 2 wavelength ranges, $1.4-2.5 \mu \mathrm{m}$ and $2.8-4 \mu \mathrm{m}$, each with a linear InSb array of 12 pixels (Noda et al. 1996). The spectral resolution was $\sim 0.1 \mu \mathrm{m}$ for point sources. The NIRS has a $8^{\prime} \times 8^{\prime}$ entrance aperture in the focal plane of the IRTS. Its point-source sensitivity was limited by confusion in densely populated area; otherwise in general the limits for detection were $K_{\text {lim }} \sim 8$ and $L_{\text {lim }}^{\prime} \sim 7$. However, in some areas close to the Galactic 


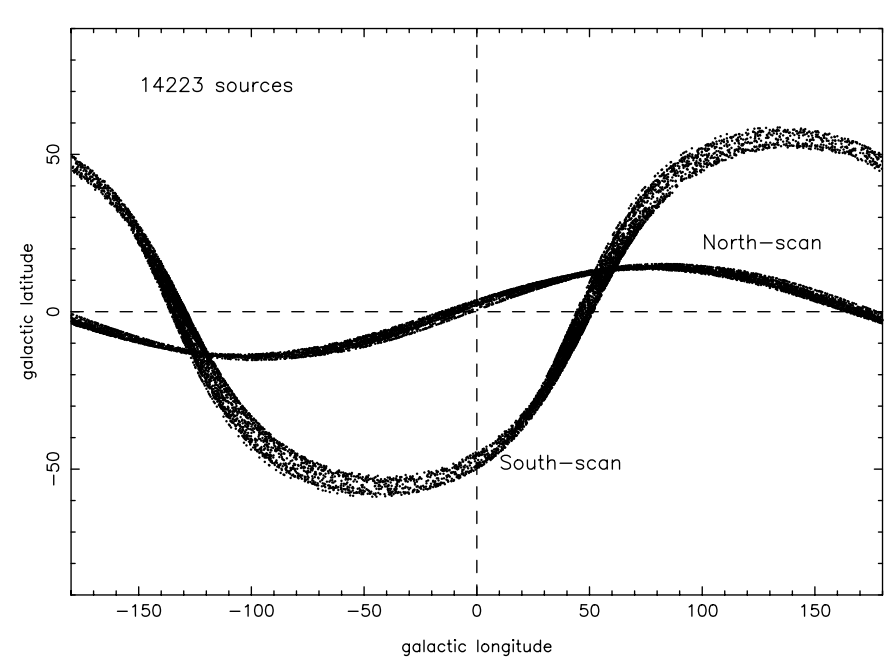

Fig. 1. Distribution of the 14223 NIRS/IRTS sources on the sky. The strip at low inclination over the Galactic Plane is refered to as the North-scan, and the other one as the South-scan.

Plane, the large galactic background obliged to operate the detectors at a reduced sensitivity $\left(K_{\lim } \sim 6\right.$ and $\left.L_{\lim }^{\prime} \sim 5\right)$.

\subsection{Data processing}

We use the 2002 data release described in Yamamura et al. $(2003)^{1}$. To extract the point source spectra, the data relevant to each source within one (North or South) scan have been combined. Only sources observed at least twice with a clear detection were kept. Many low-quality sources and sources with only one observation that appeared in the preliminary release have thus been rejected in this version. The flux calibration has been derived from standards in Cohen et al. (1999) that have been well observed by the IRTS. We believe that this release is of better quality in terms of source (catalogue entry) reliability and of photometry. In the overlap region between the North and the South scans, we find 72 duplicates; we decided to keep only the entries with the best quality. The distribution of the 14223 resulting sources on the sky is shown in Fig. 1.

We estimate the $K$ and $L^{\prime}$ magnitudes by integrating the spectra over several channels around the nominal wavelengths (2.19 and $3.78 \mu \mathrm{m})$. The flux from each channel has been weighted by the convolution between the NIRS band profile and the $K / L^{\prime}$ band profile corresponding to the ESO photometric system.

This step could be performed in $K$ and $L^{\prime}$ for 8879 NIRS point sources. We checked the quality of the photometry by comparing our results on ESO standard stars with Bouchet et al. (1991), and found the agreement to better than $0.01 \mathrm{mag}$. in $K$ (6 stars) and 0.04 mag. in $L^{\prime}$ (5 stars). The NIRS source counts in $K$ and $L^{\prime}$ are presented in Fig. 2. The distributions are linear until $K \sim 4$ and $L^{\prime} \sim 3$, and then from $K \sim 4.5$ to 6 and from $L^{\prime} \sim 4$ to 5.5. The corresponding breaks in the slopes of

\footnotetext{
1 The IRTS data and explanatories are available via DARTS archive; URL: http: //www . darts.isas . ac.jp/
}
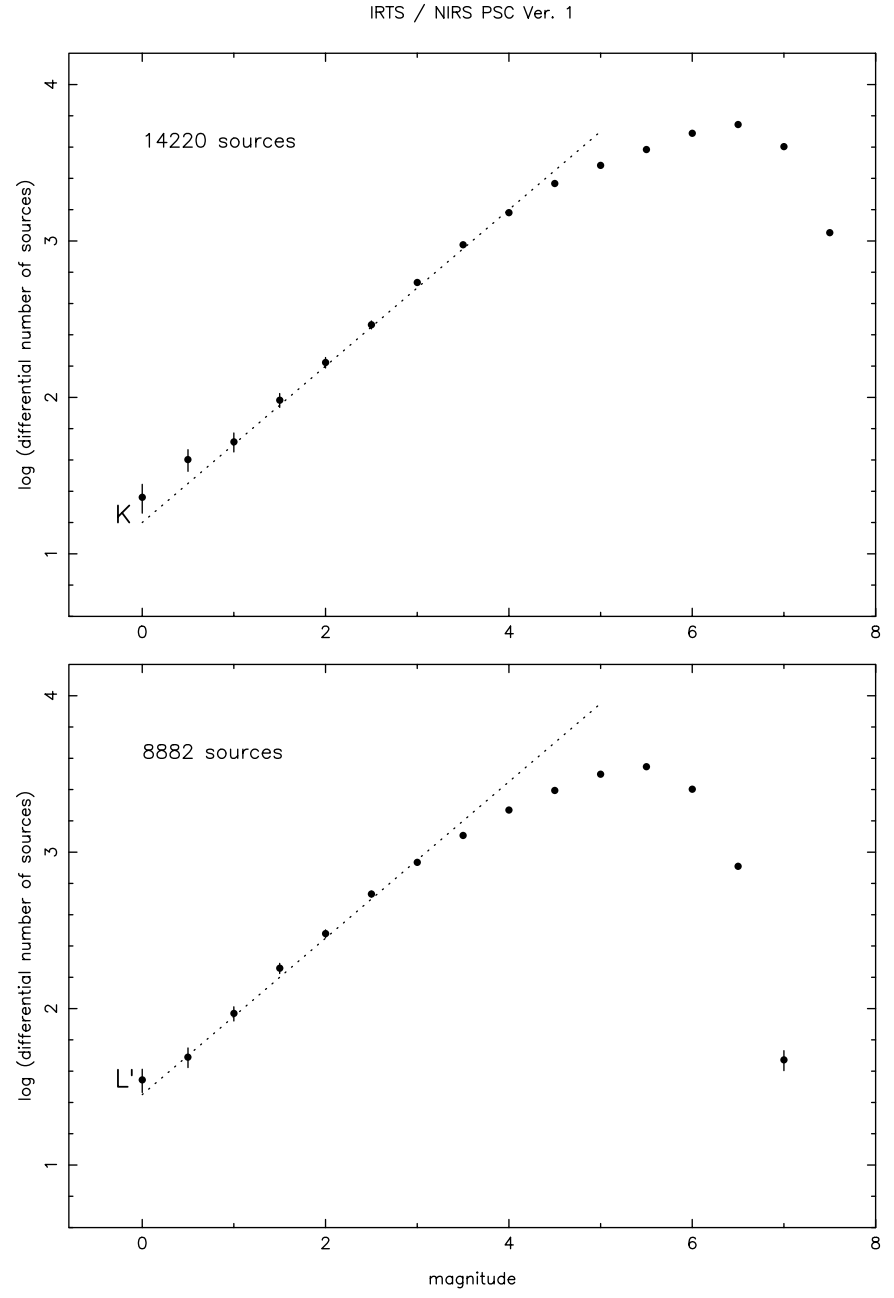

Fig. 2. NIRS source counts per unit magnitude in $K$ and $L^{\prime}$. The total number of sources in $K$ is 14220 , and in $L^{\prime}, 8882$. The error bars are estimated assuming Poisson statistics. The dotted lines are straight lines of slope $0.5 \mathrm{mag}^{-1}$.

the distributions are the effect of fewer sources being detected close to the Galactic Plane, around $l^{\mathrm{II}}=-11^{\circ}$ and $49^{\circ}$, because of the higher galactic background and of the confusion due to the higher source density (see also Sect. 4.1). The peaks of the distributions are obtained at $K \sim 6.5$ and $L^{\prime} \sim 5.5$.

\subsection{Selection of the sources}

As in Paper I, for the present analysis we consider sources with a $K-L^{\prime}$ index larger than 0.7 (863). Among them, 790 could be associated with an IRAS source. NIRS sources may have no IRAS counterpart either because the astrometry was not accurate enough to secure the identification or because they lie in a region of the sky not covered by IRAS; also, some sources do not appear in the IRAS PSC because of low data-quality. In Paper I, we used Epchtein's method to sort C-rich stars and O-rich ones (Epchtein et al. 1987). To apply this method requires the knowledge of the $K-L^{\prime}$ and the IRAS 12-25 indices with a reasonable accuracy. The NIRS spectroscopic information allowed us to check the efficiency of this method. 
Table 1. Definition of the indices.

\begin{tabular}{lcccl}
\hline \hline & Centre & \multicolumn{2}{l}{ Continuum } & Comments \\
\hline$I(1.9)$ & 1.95 & 1.74 & 2.16 & $\mathrm{H}_{2} \mathrm{O} /$ O-rich \\
$I(2.8)$ & 2.91 & 2.16 & 3.32 & $\mathrm{H}_{2} \mathrm{O} /$ O-rich \\
$I(3.1)$ & 3.12 & 2.91 & 3.32 & $\mathrm{C}_{2} \mathrm{H}_{2}+\mathrm{HCN} /$ C-rich \\
$I(3.3)$ & 3.32 & 3.12 & 3.52 & $\mathrm{UIB} / 3.3$ emission sources \\
\hline
\end{tabular}

The level of contamination by non-AGB sources was estimated to be less than 5\%. On the other hand, this method requires that the NIRS/IRAS cross-identification is correct. It is feasible to check sources individually in a small sample of 100-150 objects, but difficult in a larger one. Finally we want to avoid losing NIRS sources that have no IRAS counterpart with good fluxes at 12 and $25 \mu \mathrm{m}$. As the NIRS spectra provide the necessary information to classify sources (Yamamura et al. 1997), we can use them directly without resorting to the IRAS data. Such an approach has the advantage that it can be applied to all the 863 sources and that it avoids the ambiguities inherent to the cross-identification processes. Of course, the IRAS data can still be used to perform a posteriori checks.

We define indices that are designed to facilitate the identification of AGB stars and their chemical identification (C-rich/O-rich). The NIRS wavelength range covers several molecular absorption bands which can be used for that purpose, among which are: (i) a water band at $1.9 \mu \mathrm{m}$, (ii) a band at $3.1 \mu \mathrm{m}$ due to a blend of $\mathrm{C}_{2} \mathrm{H}_{2}$ and $\mathrm{HCN}$. The first band characterizes unambiguously $\mathrm{O}$-rich stars and the second one $\mathrm{C}$-rich ones. We construct the indices by estimating the ratio of the intensity around the centre of the features to the sum of the intensities taken on each side (i.e. on the continuum). The details are given in Table 1. The intensities are as given in the NIRS data-release, i.e. they are expressed in Jy, and summed over two pixels. An index around 0.5 points to a flat spectrum, whereas a value lower than 0.5 points to an absorption feature and a value higher than 0.5 to an emission feature. These NIRS indices should be used with care because, although the absolute wavelength calibration is excellent (Murakami et al. 2003), the spectrum of a point source moved along the detector array depending on its centering inside the entrance aperture of the spectrometer. This effect is of the order of $0.025 \mu \mathrm{m} \mathrm{rms}$, and could affect the measured values of the indices.

A diagramme representing $I(1.9)$ versus $I(3.1)$ is presented in Fig. 3. One sees two well-defined strips around $I(3.1)=0.5$ and $I(1.9)=0.5$, which merge at $(0.5,0.5)$. The first one is produced by O-rich sources and the second one is mainly due to C-rich stars. Some points are scattered; this is in part due to sources with low signal-to-noise ratio spectra but also to peculiar objects, like emission-line sources. Sources in the region labelled "O" in Fig. 3 can be safely identified as O-rich sources. The region labelled " $\mathrm{C}$ " contains mainly $\mathrm{C}$-rich stars, but also a few sources with the emission band at $3.3 \mu \mathrm{m}$ which is ascribed to PAHs. This emission raises the "continuum" at $3.3 \mu \mathrm{m}$ which, in turn, mimics an absorption at $3.1 \mu \mathrm{m}$. In order to

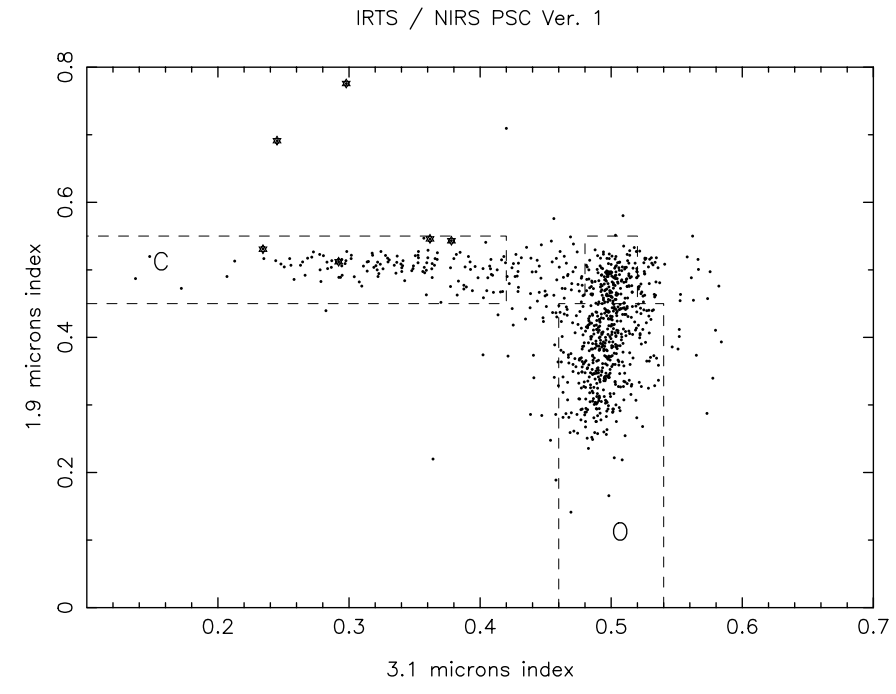

Fig. 3. $I(1.9)$ versus $I(3.1)$. The dotted lines define area discussed in the text: "C" for carbon-rich region and "O" for oxygen-rich region. The stars $(\star)$ represent sources with a strong emission band at $3.3 \mu \mathrm{m}$. One of them, with an intense $P_{\alpha}$ emission $(I(1.9) \sim 1.25)$, is out of the figure.

improve the identification of $\mathrm{C}$-rich stars in the region $\mathrm{C}$, we have constructed a third index, $I(3.3)$, designed to identify sources with PAH emission. Sources with $I(3.3)>0.75$ are represented with a “ $\star$ ” in Fig. 3 and following, and are listed in Table 2. There are several carbon stars with $0.42<I(3.1)<$ 0.48 and $0.45<I(1.9)<0.55$, but also O-rich ones.

The area around $(0.5,0.5)$ is densely populated and contains many O-rich sources with a weak absorption band at $1.9 \mu \mathrm{m}$. To help identify them we have used a fourth index, $I(2.8)$, designed to identify sources with the water absorption band at $2.8 \mu \mathrm{m}$. The definition of an index for this band is not easy because this water band is wide and merges with the CO band at $2.3 \mu \mathrm{m}$ and because the NIRS spectra present a gap between 2.5 and $2.8 \mu \mathrm{m}$. Also we wanted to avoid taking the continuum at $3.1 \mu \mathrm{m}$ because of the absorption band found in carbon stars. As the continuum points are widely separated, this index is also sensitive to the general curvature of the spectra. A diagramme representing $I(1.9)$ versus $I(2.8)$ is shown in Fig. 4. One sees that the 2 indices correlate well. We noted that many stars that barely show the water absorption band at $1.9 \mu \mathrm{m}$ still show an absorption at $2.8 \mu \mathrm{m}$ extending up to $3.5 \mu \mathrm{m}$ (see e.g. the spectrum of S Cas in the Fig. 4 of Paper I and other IRTS spectra in Matsuura et al. 1999). This means that $I(2.8)$ can be used to identify O-rich stars that have a weak $1.9 \mu \mathrm{m}$ band. In Fig. 5 we present $I(2.8)$ as a function of $I(3.1)$. This diagramme is similar to the one presented in Fig. 3. We also see 2 strips defined by $\mathrm{C}$-rich and O-rich sources. In addition the sources with the 3.3 emission-band are found along a line intermediate between these 2 strips. The scatter is larger than in Fig. 3 because the $2.8 \mu \mathrm{m}$ index is defined on a wider spectral region. Nevertheless the region labelled "O" contains O-rich sources without ambiguity. The region labelled " $\mathrm{C}$ " contains a majority of C-rich sources. 
Table 2. Sources with a strong emission band at $3.3 \mu \mathrm{m}(I(3.3)>0.75)$.

\begin{tabular}{lrrrrrlll}
\hline \hline NIRS name & $l^{\text {II }}$ & $b^{\text {II }}$ & \multicolumn{1}{l}{$H$} & $K$ & $L^{\prime}$ & $\begin{array}{l}\text { Association } \\
\text { with IRAS }\end{array}$ & Remarks \\
\hline $05281+3411 \mathrm{~N}$ & 173.92 & 0.28 & 7.00 & 6.45 & 4.23 & $05281+3412$ & NGC 1931, open cluster \\
$17167-3852 \mathrm{~N}$ & 348.75 & -1.03 & - & 5.53 & 2.15 & $17167-3854$ & \\
$17185-3533 \mathrm{~N}$ & 351.69 & 0.57 & 5.47 & 4.61 & 2.82 & $17187-3531$ & \\
$17222-3421 \mathrm{~N}$ & 353.09 & 0.64 & 4.45 & 3.78 & 1.75 & & \\
$19201+1359 \mathrm{~S}$ & 48.98 & -0.31 & 5.12 & 4.34 & 2.32 & $19201+1400$ & \\
$19209+1410 \mathrm{~S}$ & 49.23 & -0.40 & 4.49 & 4.08 & 2.91 & $19207+1410$ & GAL 049.20-00.35, HII region \\
$19213+1422 \mathrm{~S}$ & 49.45 & -0.38 & - & 5.46 & 1.52 & $19213+1424$ & W51 North \\
\hline
\end{tabular}

IRTS / NIRS PSC Ver. 1

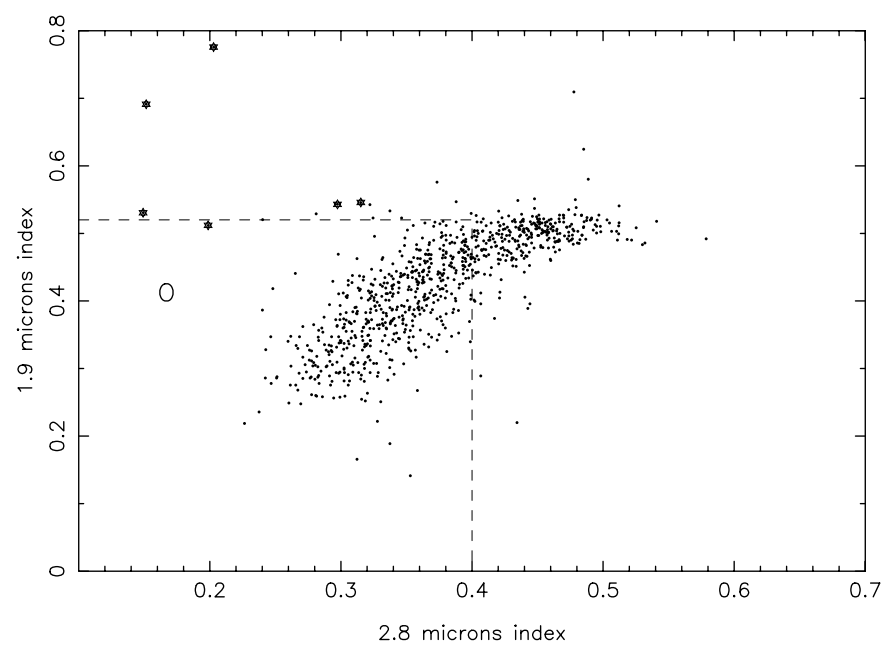

Fig. 4. $I(1.9)$ versus $I(2.8)$. The rest is as in Fig. 3.

These remarks have led us to define criteria which, using our sample, allow us to find reliably O-rich and C-rich sources.

O-rich sources:

(i) $I(1.9) \leq 0.45$ and $0.46 \leq I(3.1) \leq 0.54$, or

(ii) $0.45 \leq I(1.9) \leq 0.55$ and $0.48 \leq I(3.1) \leq 0.52$ and $I(2.8) \leq 0.40$.

C-rich sources:

$$
\begin{aligned}
& I(3.1) \leq 0.42 \text { and } 0.45 \leq I(1.9) \leq 0.55 \\
& \text { and } 0.40 \leq I(2.8) \leq 0.50 .
\end{aligned}
$$

For the sources that do not fulfill one of these criteria we determined their type (C, O, dubious/other) by visual inspection. We also checked individually all C-rich sources. Tanaka et al. (in preparation) have developed a similar, but slightly different, method and obtained consistent results.

\subsection{Description of the sample}

At the first stage with these criteria, we obtained $110 \mathrm{C}$-rich stars, 489 O-rich stars and 264 objects, which had to be looked at individually, out of 863 sources. After visual inspection, we kept 126 C-rich sources and 563 O-rich ones. Most of the remaining sources are found close to the Galactic Plane and/or have a spectrum with a low signal-to-noise ratio. Some could

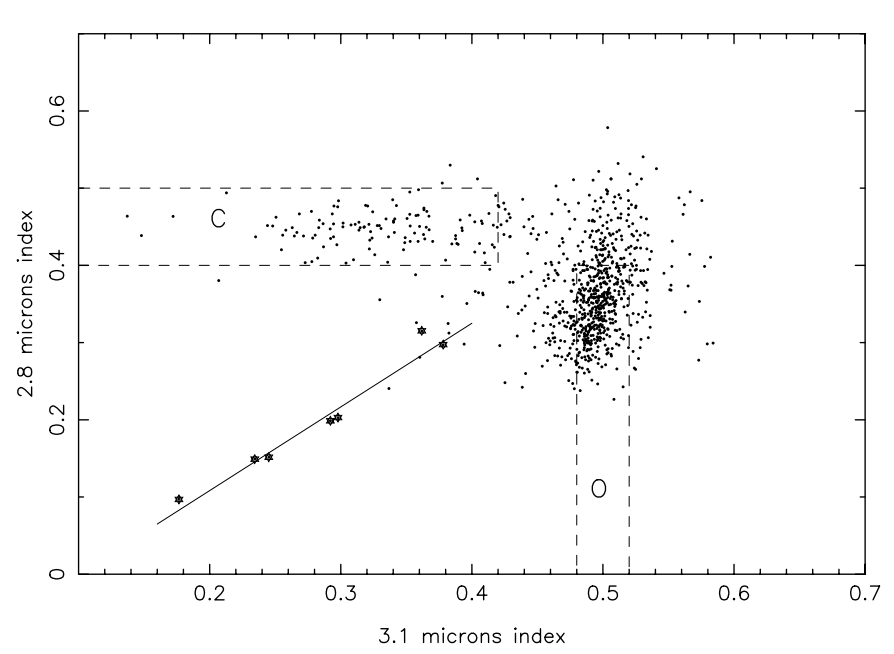

Fig. 5. $I(2.8)$ versus $I(3.1)$. The sources with a strong emission band at $3.3 \mu \mathrm{m}$ are found along the full line. The rest is as in Fig. 3.

be identified in Simbad as T Tauri stars or early-type emission line sources.

Some objects (35) which were listed among the 126 AGB sources in Paper I do not appear in the present sample because they were rejected at the level of the 2002 IRTS data-release as they did not satisfy the quality level criteria for this release.

\section{Results}

We apply the same approach as in Paper I to derive distances, corrected for interstellar extinction. We use the $K$ magnitude and a bolometric correction based on the $K-L^{\prime}$ index, and assume a bolometric magnitude corresponding to $8000 L_{\odot}$. As the fluxes are better determined we expect that the distances are more reliable, but of course with the same limitations as before, i.e. estimates of the distances to individual sources could be uncertain by a factor of almost 2 , because of the source variability and because of the assumption that all sources have the same luminosity.

The mass loss rates are determined also following the approach of Paper I. For C-rich stars we use the relation between $K-L^{\prime}$ and $\dot{M}$ obtained by Le Bertre (1997), and for O-rich stars, the one obtained by Le Bertre \& Winters (1998). These relations are distance independent. However, the 
Table 3. Properties of the 20 C-rich sources with $\dot{M} \geq 10^{-6} M_{\odot} \mathrm{yr}^{-1}$. The complete table with the 126 C-rich sources is available at CDS.

\begin{tabular}{llllllrrc}
\hline \hline NIRS name & $\begin{array}{l}\text { Association } \\
\text { with IRAS }\end{array}$ & $n$ & $K$ & $K-L^{\prime}$ & $\begin{array}{c}d_{\mathrm{Sun}} \\
\mathrm{pc}\end{array}$ & $\begin{array}{r}z \\
\mathrm{pc}\end{array}$ & $\begin{array}{r}d_{\mathrm{GC}} \\
\mathrm{kpc}\end{array}$ & $\begin{array}{r}\log \dot{M}_{-6} \\
10^{-6} M_{\odot} \mathrm{yr}^{-1}\end{array}$ \\
\hline $02596+6638 \mathrm{~N}$ & $02596+6639$ & 3 & 4.561 & 2.281 & 2490. & 314. & 10.41 & 0.282 \\
$03562+5911 \mathrm{~N}$ & $03561+5912$ & 2 & 6.114 & 2.376 & 4749. & 399. & 12.67 & 0.336 \\
$04393+4905 \mathrm{~N}$ & $04391+4904$ & 2 & 5.764 & 2.143 & 4701. & 170. & 12.95 & 0.152 \\
$04447+4951 \mathrm{~N}$ & $04449+4951$ & 4 & 4.656 & 2.774 & 1803. & 102. & 10.18 & 0.569 \\
$05102+4520 \mathrm{~N}$ & $05106+4520$ & 2 & 5.984 & 2.713 & 3481. & 232. & 11.86 & 0.533 \\
$05151+3511 \mathrm{~N}$ & $05149+3511$ & 2 & 3.610 & 1.974 & 1764. & -42. & 10.25 & 0.034 \\
$05537+1158 \mathrm{~N}$ & $05537+1157$ & 5 & 5.941 & 2.034 & 5242. & -585. & 13.58 & 0.101 \\
$11318-7256 \mathrm{~N}$ & $11318-7256$ & 6 & 1.323 & 1.938 & 632. & -123. & 8.23 & 0.038 \\
$17131-3906 \mathrm{~N}$ & $17130-3907$ & 2 & 4.446 & 2.509 & 2008. & -21. & 6.55 & 0.378 \\
$20547+6404 \mathrm{~N}$ & $20546+6405$ & 2 & 4.700 & 2.311 & 2593. & 548. & 9.30 & 0.310 \\
$07027-1456 \mathrm{~S}$ & $07028-1456$ & 3 & 4.383 & 2.526 & 1913. & -128. & 9.88 & 0.430 \\
$07036-2221 \mathrm{~S}$ & $07036-2220$ & 3 & 6.902 & 3.729 & 2485. & -305. & 10.13 & 0.983 \\
$07099-2012 \mathrm{~S}$ & $07098-2012$ & 9 & 4.031 & 2.893 & 1236. & -103. & 9.29 & 0.636 \\
$07118-1023 \mathrm{~S}$ & $07118-1022$ & 5 & 5.419 & 2.252 & 3813. & 15. & 11.52 & 0.198 \\
$07216-1245 \mathrm{~S}$ & $07217-1246$ & 3 & 4.194 & 2.903 & 1324. & 28. & 9.44 & 0.633 \\
$07225-0538 \mathrm{~S}$ & $07227-0537$ & 4 & 6.264 & 2.228 & 5683. & 476. & 13.27 & 0.236 \\
$07336-1005 \mathrm{~S}$ & $07336-1006$ & 6 & 7.055 & 3.839 & 2458. & 217. & 10.32 & 1.018 \\
$19028+2018 \mathrm{~S}$ & $19029+2017$ & 3 & 4.654 & 2.927 & 1606. & 176. & 7.64 & 0.650 \\
$19109+1156 \mathrm{~S}$ & $19108+1155$ & 2 & 3.836 & 2.055 & 1902. & 24. & 7.31 & 0.063 \\
$19248+0656 \mathrm{~S}$ & $19248+0658$ & 3 & 3.476 & 2.429 & 1354. & -110. & 7.57 & 0.371 \\
\hline
\end{tabular}

distances are taken into account indirectly through the correction for interstellar reddening.

The results are given in Table 3 for C-rich sources and in Table 4 for O-rich ones (only those with mass loss rates above $10^{-6} M_{\odot} \mathrm{yr}^{-1}$ are listed in the printed version). The first column gives the NIRS name corresponding the 2002 data-release and built from the 1950.0 coordinates and a letter, $\mathrm{N}$ or $\mathrm{S}$, refering to the North or South-scan. The second column gives the IRAS name when a reasonable association could be obtained; some may still be incorrect. The third column gives the number of events used to derive the NIRS spectra (this is an indication of quality). The fourth column gives the $K$ magnitude, and the fifth one, the $K-L^{\prime}$ index, both derived from the NIRS spectra in the ESO photometric system. The 6th, 7th, and 8th columns give the distance to the Sun $\left(d_{\text {Sun }}\right)$, the distance to the Galactic Plane $(z)$ and the distance to the Galactic Center projected on the Galactic Plane $\left(d_{\mathrm{GC}}\right)$. Finally the last column give the logarithm of the mass loss rate expressed in $10^{-6} M_{\odot} \mathrm{yr}^{-1}$.

\section{Discussion}

The larger size of the sample allows us to refine the analysis of the space distribution of mass losing AGB stars and of the relative contributions of $\mathrm{C}$ and O-rich sources to the replenishment of the ISM.

\subsection{Space distribution of the sources}

In Fig. 6 we show the projection of our sample of mass-losing AGB stars on the Galactic Plane (GP). Sources are found preferentially at the galactic longitudes corresponding to the intersections of the IRTS scans with the GP (Fig. 1). However, for $l^{\mathrm{II}}=-10^{\circ}$ and $+49^{\circ}$, one notes a lack of sources beyond $3 \mathrm{kpc}$.
This effect comes from the reduced sensitivity at which the detectors were operated, and from the confusion due to the higher stellar density close to the GP. It is the same effect responsible for the breaks observed in Fig. 2 at $K \sim 4$ and $L^{\prime} \sim 3$. Also, AGB stars at low galactic latitudes may have larger mean luminosities than AGB stars at high galactic latitude (e.g. Kastner et al. 1993). Therefore, it could be that we are underestimating the distance to these sources with the implication that they would look "closer" to the Sun in Fig. 6. However, the lack of sources with $K>4$ and $L^{\prime}>3$ at low $b^{\mathrm{II}}$ is general, not specific to AGB stars in our sample (cf. Fig. 2).

As in Paper I, we find that most mass-losing carbon-stars are located preferentially in the exterior of our Galaxy. The new analysis with a much larger sample clearly confirms this trend, as is shown in the lower panel of Fig. 7. Only one carbon star is found at $r<6 \mathrm{kpc}$.

This carbon star, NIRS 20229-1641, shows the same features as the other carbon stars in our sample (Fig. 8) and there is no ambiguity on its carbon-rich classification. In fact its distance to the GP is $\sim 3.4 \mathrm{kpc}$ and it probably belongs to the halo of our Galaxy. From its direction on the sky, it does not seem to be related to the Sgr dwarf galaxy (Ibata et al. 1995). Another source, NIRS 07001-2412 (三 IRAS 07000-2411), is located at $-1.1 \mathrm{kpc}$ from the GP and probably also belongs to the halo. Its NIRS spectrum is noisy but the $3.1 \mu \mathrm{m}$ absorption feature is clearly present (Fig. 9); it has already been reported as C-rich by Stephenson (1989; CGCS 1517). For both sources a radial velocity would be useful to clarify their nature.

The increasing ratio of C-rich to O-rich AGB stars with increasing galactocentric distance has already been inferred by Jura \& Kleinmann (1990) who showed that the space distribution of very dusty carbon stars is almost constant within $1 \mathrm{kpc}$ of the Sun, in contrast to the distribution of mass-losing 
Table 4. Properties of the 56 O-rich sources with $\dot{M} \geq 10^{-6} M_{\odot} \mathrm{yr}^{-1}$. The complete table with the 563 O-rich sources is available at CDS.

\begin{tabular}{|c|c|c|c|c|c|c|c|c|}
\hline NIRS name & $\begin{array}{l}\text { Association } \\
\text { with IRAS }\end{array}$ & $n$ & $K$ & $K-L^{\prime}$ & $\begin{array}{c}d_{\text {Sun }} \\
\text { pc }\end{array}$ & $\begin{array}{r}z \\
\mathrm{pc}\end{array}$ & $\begin{array}{l}d_{\mathrm{GC}} \\
\mathrm{kpc}\end{array}$ & $\begin{array}{c}\log \dot{M}_{-6} \\
10^{-6} M_{\odot} \mathrm{yr}^{-1}\end{array}$ \\
\hline $01161+7221 \mathrm{~N}$ & $01159+7220$ & 3 & 1.765 & 1.291 & 774. & 133. & 8.96 & 0.086 \\
\hline $12593-7356 \mathrm{~N}$ & $12593-7355$ & 2 & 4.655 & 1.293 & 2918. & -574 & 7.31 & 0.078 \\
\hline $14018-7319 \mathrm{~N}$ & $14014-7319$ & 3 & 4.656 & 1.266 & 2919. & -580 & 7.09 & 0.031 \\
\hline $14322-7156 \mathrm{~N}$ & $14319-7154$ & 2 & 5.526 & 1.621 & 4353. & -826 & 6.55 & 0.523 \\
\hline $15129-6801 \mathrm{~N}$ & $15129-6800$ & 2 & 5.341 & 1.332 & 3980. & -629 & 6.30 & 0.130 \\
\hline $15155-6529 \mathrm{~N}$ & $15155-6531$ & 2 & 3.646 & 1.359 & 1817. & -224 & 7.27 & 0.164 \\
\hline $15232-6715 \mathrm{~N}$ & $15232-6716$ & 3 & 5.311 & 1.539 & 3924. & -613 & 6.24 & 0.421 \\
\hline $15433-6325 \mathrm{~N}$ & $15431-6324$ & 2 & 5.717 & 1.716 & 4697. & -582 & 5.68 & 0.602 \\
\hline $16030-5928 \mathrm{~N}$ & $16030-5928$ & 3 & 4.589 & 1.915 & 2775. & -271 & 6.42 & 0.770 \\
\hline $16153-5613 \mathrm{~N}$ & $16156-5613$ & 2 & 3.435 & 1.315 & 1634. & -124 & 7.15 & 0.067 \\
\hline $16196-5647 \mathrm{~N}$ & $16196-5647$ & 4 & 4.887 & 1.344 & 3169. & -286 & 6.02 & 0.101 \\
\hline $16407-5127 \mathrm{~N}$ & $16409-5128$ & 2 & 4.497 & 1.517 & 2622. & -174 . & 6.23 & 0.337 \\
\hline $16414-4940 \mathrm{~N}$ & $16414-4941$ & 5 & 4.065 & 1.465 & 2139. & -101 & 6.60 & 0.255 \\
\hline $16549-4540 \mathrm{~N}$ & $16551-4541$ & 2 & 3.899 & 1.566 & 1971. & -65 & 6.67 & 0.381 \\
\hline $17025-4719 \mathrm{~N}$ & $17025-4719$ & 3 & 3.186 & 1.628 & 1457. & -100 & 7.15 & 0.502 \\
\hline $17108-3753 \mathrm{~N}$ & $17109-3753$ & 8 & 4.101 & 1.604 & 2102. & 17. & 6.45 & 0.372 \\
\hline $17146-4158 \mathrm{~N}$ & $17146-4158$ & 2 & 4.664 & 1.685 & 2771. & -120 & 5.85 & 0.501 \\
\hline $17172-3756 \mathrm{~N}$ & & 3 & 4.335 & 1.379 & 2318. & -23 & 6.23 & 0.003 \\
\hline $17210-3318 \mathrm{~N}$ & $17209-3318$ & 3 & 4.514 & 2.685 & 1813. & 46. & 6.70 & 1.191 \\
\hline $17210-3127 \mathrm{~N}$ & $17209-3126$ & 2 & 4.321 & 2.078 & 2384. & 103. & 6.13 & 0.858 \\
\hline $17215-3548 \mathrm{~N}$ & $17215-3551$ & 2 & 3.846 & 1.375 & 1879. & -2 & 6.65 & 0.039 \\
\hline $17240-3448 \mathrm{~N}$ & $17240-3449$ & 5 & 3.586 & 1.875 & 1687. & 2. & 6.83 & 0.689 \\
\hline $17250-3408 \mathrm{~N}$ & $17250-3408$ & 4 & 3.993 & 1.565 & 2003. & 10. & 6.51 & 0.324 \\
\hline $17322-2422 \mathrm{~N}$ & $17323-2424$ & 3 & 5.909 & 1.873 & 4986. & 378. & 3.54 & 0.706 \\
\hline $17355-3155 \mathrm{~N}$ & $17354-3155$ & 7 & 3.383 & 2.098 & 1552. & -9 & 6.95 & 0.876 \\
\hline $17366-2318 \mathrm{~N}$ & $17367-2319$ & 6 & 3.130 & 1.457 & 1421. & 101. & 7.09 & 0.291 \\
\hline $17398-2100 \mathrm{~N}$ & 17396-2059 & 2 & 6.084 & 1.573 & 5427. & 441. & 3.18 & 0.402 \\
\hline $17503-1517 \mathrm{~N}$ & $17502-1519$ & 6 & 5.191 & 1.409 & 3643. & 348. & 5.02 & 0.203 \\
\hline $18077-0237 \mathrm{~N}$ & $18078-0238$ & 4 & 7.064 & 2.720 & 5688. & 783. & 4.22 & 1.223 \\
\hline $18201+0636 \mathrm{~N}$ & $18202+0636$ & 4 & 4.238 & 1.298 & 2397. & 392. & 6.72 & 0.075 \\
\hline $19106+4148 \mathrm{~N}$ & $19105+4148$ & 8 & 5.320 & 1.434 & 3979. & 973. & 8.25 & 0.304 \\
\hline $19564+5449 \mathrm{~N}$ & & 2 & 6.088 & 1.251 & 5663. & 1289. & 10.01 & 0.013 \\
\hline $20532+6606 \mathrm{~N}$ & $20535+6606$ & 2 & 5.793 & 1.348 & 4948. & 1163. & 10.61 & 0.178 \\
\hline $23555+7233 \mathrm{~N}$ & $23551+7233$ & 4 & 5.557 & 1.311 & 4423. & 798. & 11.27 & 0.110 \\
\hline $06455-2104 \mathrm{~S}$ & $06455-2107$ & 4 & 6.519 & 1.544 & 6889. & -1226 & 13.78 & 0.439 \\
\hline 07179-1312 S & 07180-1314 & 2 & 3.895 & 1.717 & 1981. & 8. & 9.93 & 0.566 \\
\hline $18076+3444 \mathrm{~S}$ & $18076+3445$ & 3 & 4.102 & 1.731 & 2282. & 898. & 7.72 & 0.650 \\
\hline $18512+2029 \mathrm{~S}$ & $18512+2029$ & 2 & 4.988 & 1.993 & 3382. & 517. & 6.94 & 0.845 \\
\hline $19116+1939 \mathrm{~S}$ & $19117+1940$ & 4 & 4.896 & 1.319 & 3166. & 229. & 7.07 & 0.044 \\
\hline $19126+1051 \mathrm{~S}$ & & 2 & 4.263 & 1.729 & 2255. & -7 & 7.10 & 0.512 \\
\hline $19137+0931 \mathrm{~S}$ & $19137+0934$ & 5 & 3.597 & 1.957 & 1721. & -32 & 7.37 & 0.777 \\
\hline $19143+1817 \mathrm{~S}$ & $19143+1817$ & 5 & 4.258 & 1.311 & 2348. & 121. & 7.30 & 0.015 \\
\hline $19170+0813 \mathrm{~S}$ & $19168+0814$ & 3 & 4.295 & 1.575 & 2367. & -98 & 6.98 & 0.393 \\
\hline $19192+0921 \mathrm{~S}$ & $19192+0922$ & 3 & 3.755 & 2.632 & 1325. & -54 & 7.62 & 1.182 \\
\hline $19224+0732 \mathrm{~S}$ & $19224+0732$ & 2 & 3.908 & 1.313 & 2018. & -136 & 7.18 & 0.047 \\
\hline $19282+1420 \mathrm{~S}$ & $19283+1421$ & 6 & 4.918 & 1.703 & 3092. & -100 & 6.94 & 0.508 \\
\hline $19292+0502 \mathrm{~S}$ & $19291+0502$ & 2 & 4.754 & 1.454 & 3011. & -344 & 6.60 & 0.293 \\
\hline $19302+0231 \mathrm{~S}$ & $19302+0228$ & 2 & 5.786 & 1.685 & 4867. & -675 & 5.72 & 0.577 \\
\hline $19311+1131 \mathrm{~S}$ & $19312+1130$ & 10 & 5.150 & 1.451 & 3538. & -238 & 6.68 & 0.242 \\
\hline $19344+0914 \mathrm{~S}$ & $19343+0912$ & 2 & 4.684 & 1.386 & 2903. & -286 & 6.84 & 0.183 \\
\hline $19372+0549 \mathrm{~S}$ & $19374+0550$ & 8 & 2.640 & 1.386 & 1150. & -159 & 7.72 & 0.220 \\
\hline $19411+0336 \mathrm{~S}$ & $19412+0337$ & 5 & 3.089 & 1.745 & 1417. & -242 & 7.53 & 0.647 \\
\hline $19433-0157 \mathrm{~S}$ & $19433-0158$ & 3 & 5.244 & 1.968 & 3833. & -860 & 5.99 & 0.835 \\
\hline $19453+0355 \mathrm{~S}$ & $19454+0355$ & 8 & 3.376 & 1.346 & 1618. & -298 & 7.42 & 0.164 \\
\hline $20077-0624 \mathrm{~S}$ & $20077-0625$ & 26 & 2.673 & 2.538 & 860. & -300 & 7.87 & 1.160 \\
\hline $20233-1358 \mathrm{~S}$ & 20234-1357 & 23 & 2.905 & 1.255 & 1317. & -600 & 7.52 & 0.039 \\
\hline
\end{tabular}




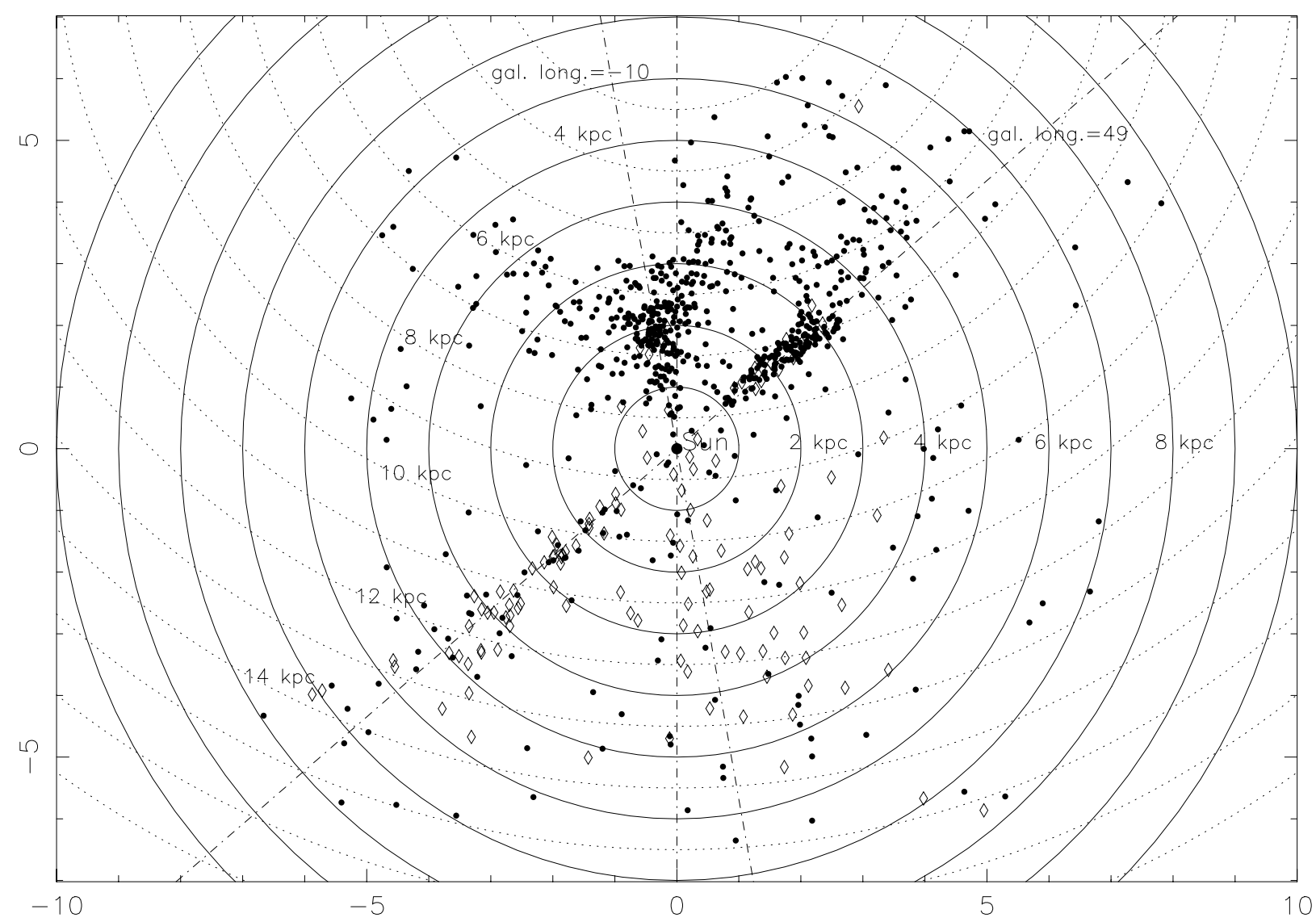

Fig. 6. Space distribution projected on the Galactic Plane. The carbon stars are represented with diamonds $(\diamond)$ and the oxygen-rich stars with $\operatorname{dots}(\bullet)$.

AGB stars which is decreasing exponentially with $d_{\mathrm{GC}}$ (Habing 1998). This result was extended to distances of $\sim 3 \mathrm{kpc}$ from the Sun by Guglielmo et al. (1993). Also, Kastner et al. (1993) found that luminous carbon stars near the Galactic Plane are located preferentially outside the solar circle.

This galactic segregation of AGB star classes is usually interpreted as an effect of the metallicity on the formation of carbon stars. Indeed the metallicity is decreasing with galactocentric distance at a rate of $\sim 0.05-0.1$ dex per kpc (Pagel 1997) and stellar evolution models of AGB stars show that a $\mathrm{C} / \mathrm{O}$ abundance ratio larger than 1 is obtained more rapidly, if at all, for low metallicity (e.g. Lattanzio \& Forestini 1999).

The lower panel of Fig. 7 shows also that most of the oxygen-rich sources in our sample are at a distance below $8 \mathrm{kpc}$ from the Galactic Center. It is noteworthy that only a few sources are found at the same galactocentric distance as the Sun $\left(8 \mathrm{kpc}<d_{\mathrm{GC}}<9 \mathrm{kpc}\right)$. This is because only a small volume of the Solar Neighborhood has been surveyed by the IRTS and because the GP at longitudes $\sim 90$ and $270^{\circ}$ has not been well sampled. This illustrates the necessity for a deeper and complete "all-sky" survey to obtain a sample representative of the AGB-star population in the solar environment.

The upper panel of Fig. 7 shows that most of the sources are at a distance between 1 and $4 \mathrm{kpc}$ from the Sun. From this histogram there is no difference between the $\mathrm{C}$-rich and the O-rich sources. There is also no obvious difference in the dependence on the distance to the GP ( $z$ ) although one notes the paucity of mass-losing carbon-stars at more than $\sim 500 \mathrm{pc}$ from the GP (see Table 6).

\subsection{Contribution to the replenishment of the ISM}

Our present selection of sources suffers from several observational biases. It is therefore difficult to infer quantitative results. However, these biases should affect equally C-rich sources and O-rich ones and we may still study the relative contributions of these 2 kinds of sources in different regions of the Galaxy.

The contributions of the 2 kinds of sources as a function of the galactocentric distance is given in Table 5, and as a function of the distance to the GP in Table 6. It appears that these contributions follow the source distribution. There is no over-representation of one category w.r.t. the other one, i.e. the sources appear to contribute proportionally to their number (Table 7).

Jura \& Kleinmann (1989) made an inventory of AGB stars losing more than $2 \times 10^{-6} M_{\odot} \mathrm{yr}^{-1}$ within $1 \mathrm{kpc}$ of the Sun; they identified 63 such sources. They find that carbon stars and O-rich ones contribute about equally to the ISM replenishment. In the range $8 \mathrm{kpc}<d_{\mathrm{GC}}<9 \mathrm{kpc}$, we find that carbon stars contribute to $\sim 0.21$ of the corresponding sample mass loss rate, and in the range $9 \mathrm{kpc}<d_{\mathrm{GC}}<10 \mathrm{kpc}$, to $\sim 0.64$ (Table 5). Our result is therefore in good agreement with that of Jura \& Kleinmann, although the samples that are analyzed are quite different. 
Table 5. Numbers of carbon-rich and oxygen-rich sources and their respective contributions to the replenishment of the ISM at different distances from the Galactic Centre.

\begin{tabular}{|c|c|c|c|c|c|c|c|c|}
\hline$d_{\mathrm{GC}}$ & $\begin{array}{r}\text { C-rich } \\
N\end{array}$ & $\sum \dot{M}_{-6}$ & $\begin{array}{r}\text { O-rich } \\
N\end{array}$ & $\sum \dot{M}_{-6}$ & $\begin{array}{r}\text { total } \\
N\end{array}$ & $\sum \dot{M}_{-6}$ & $\begin{array}{r}\text { ratio } \\
\quad N\end{array}$ & $\begin{array}{r}\mathrm{C} /(\mathrm{O}+\mathrm{C}) \\
\sum \dot{M}_{-6}\end{array}$ \\
\hline $3-4 \mathrm{kpc}$ & 0 & 0.00 & 7 & 8.36 & 7 & 8.36 & 0.00 & 0.00 \\
\hline $4-5 \mathrm{kpc}$ & 1 & 0.11 & 21 & 18.85 & 22 & 18.97 & 0.05 & 0.01 \\
\hline $5-6 \mathrm{kpc}$ & 0 & 0.00 & 85 & 28.99 & 85 & 28.99 & 0.00 & 0.00 \\
\hline 6-7 kpc & 9 & 3.49 & 193 & 112.43 & 202 & 115.92 & 0.04 & 0.03 \\
\hline $7-8 \mathrm{kpc}$ & 12 & 9.18 & 152 & 85.84 & 164 & 95.02 & 0.07 & 0.10 \\
\hline $8-9 \mathrm{kpc}$ & 8 & 1.75 & 23 & 6.55 & 31 & 8.31 & 0.26 & 0.21 \\
\hline $9-10 \mathrm{kpc}$ & 14 & 14.99 & 23 & 8.28 & 37 & 23.26 & 0.38 & 0.64 \\
\hline $10-11 \mathrm{kpc}$ & 27 & 30.53 & 19 & 6.07 & 46 & 36.59 & 0.59 & 0.83 \\
\hline $11-12 \mathrm{kpc}$ & 27 & 7.97 & 14 & 2.58 & 41 & 10.55 & 0.66 & 0.76 \\
\hline $12-13 \mathrm{kpc}$ & 19 & 5.49 & 13 & 1.67 & 32 & 7.16 & 0.59 & 0.77 \\
\hline $13-14 \mathrm{kpc}$ & 7 & 3.38 & 10 & 3.33 & 17 & 6.71 & 0.41 & 0.50 \\
\hline $14-15 \mathrm{kpc}$ & 1 & 0.12 & 3 & 0.27 & 4 & 0.39 & 0.25 & 0.31 \\
\hline $15-16 \mathrm{kpc}$ & 1 & 0.09 & 0 & 0.00 & 1 & 0.09 & 1.00 & 1.00 \\
\hline
\end{tabular}

IRTS / NIRS PSC Ver. 1
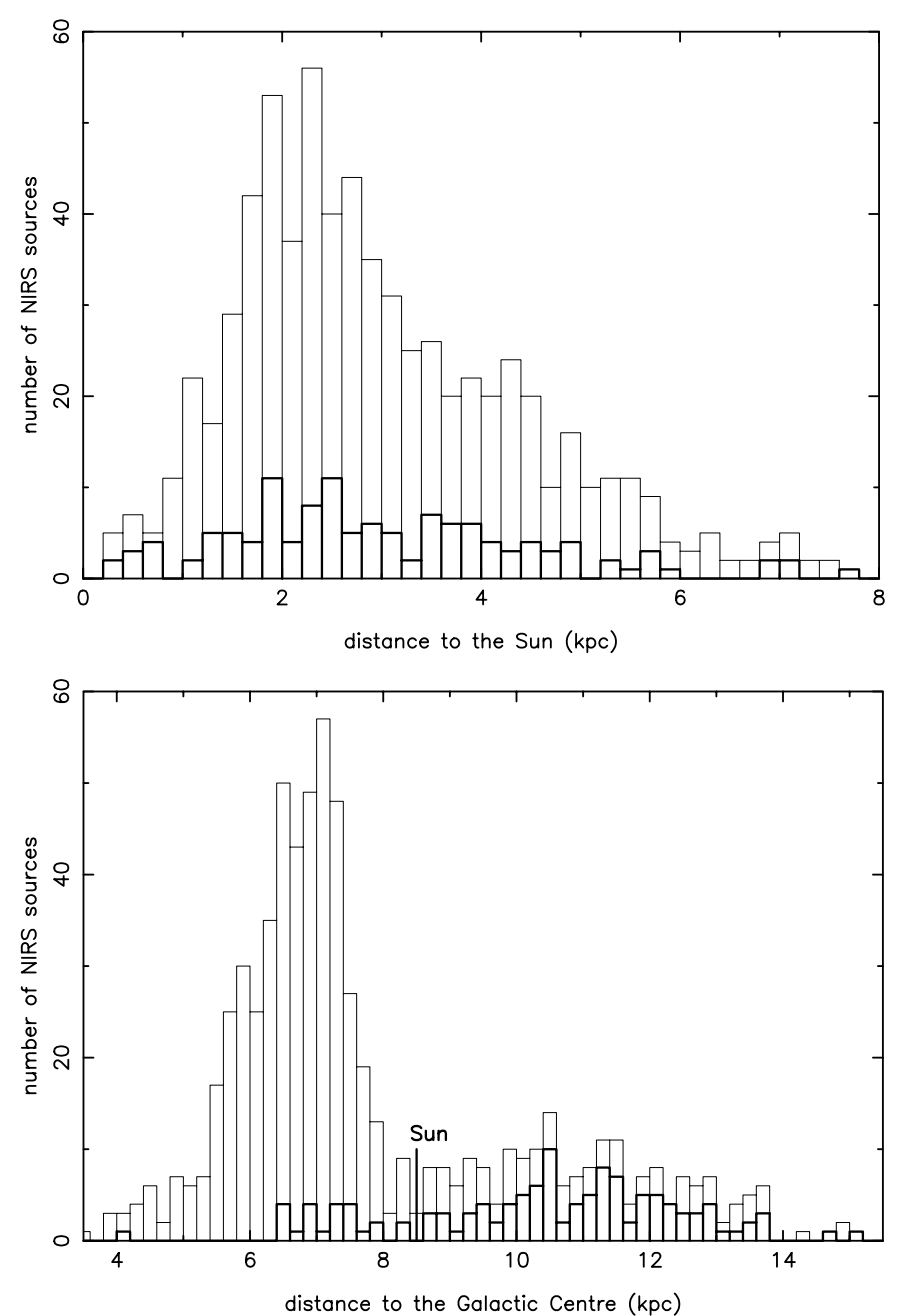

Fig. 7. Distribution through the Galaxy. The thick lines represent the carbon stars and the thin lines all the sources in the sample of masslosing AGB stars. Upper panel: as a function of the distance to the Sun $(d)$; lower panel: as a function of the distance to the Galactic Center (projected on the Galactic Plane, $r$ ).
From Table 7, one sees that about half of the contribution comes from the 71 sources $\left(\sim 10 \%\right.$ of the sample) with $10^{-6}<$ $\dot{M}<10^{-5} M_{\odot} \mathrm{yr}^{-1}$. It appears also that a few rare objects dominate the rate of ISM replenishment by mass-losing AGB stars. For instance only 5 sources out of 689 , with $\dot{M}>10^{-5} M_{\odot} \mathrm{yr}^{-1}$, contribute as much as $20 \%$ of the total (see Table 7). The dominance of this rate by a relatively small percentage was already suggested by our preliminary analysis (Paper I). The same finding was reported by Jura \& Kleinmann (1989) who noted that, in their sample of 63 AGB stars, one source, IRAS 17411-3154 (RAFGL 5379), contributes nearly $25 \%$ to the total from their sample.

However these conclusions should be handled with care as it appears that we are still limited by small number statistics. Also, in the Solar Neighborhood $\left(d_{\text {Sun }} \leq 1 \mathrm{kpc}\right)$, we detect only 3 sources with $\dot{M} \geq 10^{-6} M_{\odot} \mathrm{yr}^{-1}$, whereas Jura \& Kleinmann (1989) identified 63 such sources. Notably, one of our 3 sources (IRAS 11318-7256, CGCS 3062) is not in their list, because it lies outside the zone that they explored $\left(\delta<-33^{\circ}\right)$.

\section{Conclusion}

The NIRS data illustrates the advantage of a spectrophotometric survey, with a resolution of $\geq 40$, over classical photometric surveys which alone cannot secure any chemical identification. The ability to perform directly spectrophotometry reduces the uncertainties in the determination of stellar chemistries and avoids any risk connected to cross-identification errors.

The present work confirms the main results obtained in Paper I. With a NIRS sample that is about 5 times larger, and more reliable, we show that the replenishment of the ISM is dominated by the high mass-loss rate stars. We obtain a clearer separation of oxygen-rich and carbon-rich stars with a border at a distance from the Galactic Centre of about the solar distance. However, we note that a slightly better spectral resolution and a larger wavelength coverage (1.4 to $4.2 \mu \mathrm{m}$, with no gap around $2.7 \mu \mathrm{m}$ ) would have facilitated our study. 
IRTS / NIRS PSC Ver. 1
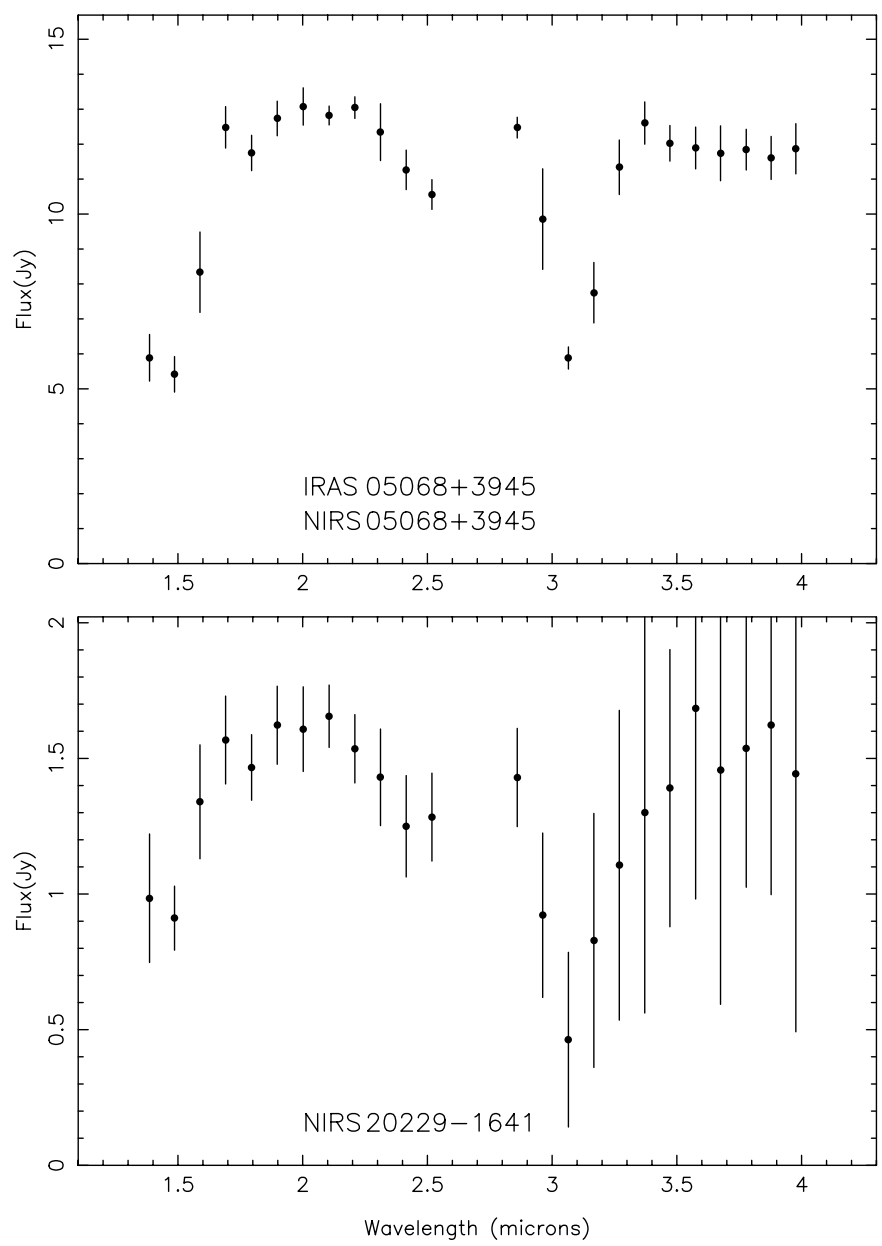

Fig. 8. Carbon star spectra. Upper panel: a carbon star (V480 Aur, CGCS 867) in the galactic anticenter direction; lower panel: an unidentifed carbon star (Sect. 4.1). Note the absorption features in common at $1.5(\mathrm{CO}), 1.8\left(\mathrm{C}_{2}\right)$ and $3.1 \mu \mathrm{m}\left(\mathrm{C}_{2} \mathrm{H}_{2}+\mathrm{HCN}\right)$.

Furthermore, our analysis is limited by the sensitivity of the NIRS to point sources, especially in the regions of large galactic background. Also the close Solar Neighborhood (8 kpc < $r_{\mathrm{GC}}<9 \mathrm{kpc}$ ) is not well sampled because only $\sim 7 \%$ of the sky was covered by the IRTS. Finally, a better spatial resolution would have limited the effects of confusion in the Galactic Plane, and simultaneously reduced the intensity of the background.

Although the sample is larger by a factor $\sim 5$, we are still limited by small number statistics. This is because the sources which are presently undergoing mass loss at a very large rate $\left(\geq 10^{-5} M_{\odot} \mathrm{yr}^{-1}\right)$ are rare, a handful in a sample of $\sim 9000$ sources detected in $L^{\prime}$.

A new spectro-photometric mission with a slightly better spectral resolution $(R \sim 100)$ and spectral coverage $(1.4$ to $4.2 \mu \mathrm{m}$ or more, without gap), and a better spatial resolution (sub-arcminute), with a complete sky coverage, such as RESPIRE (Le Bertre et al. 2002), would aid our understanding of evolved stars in our Galaxy and of their rôle in its evolution.

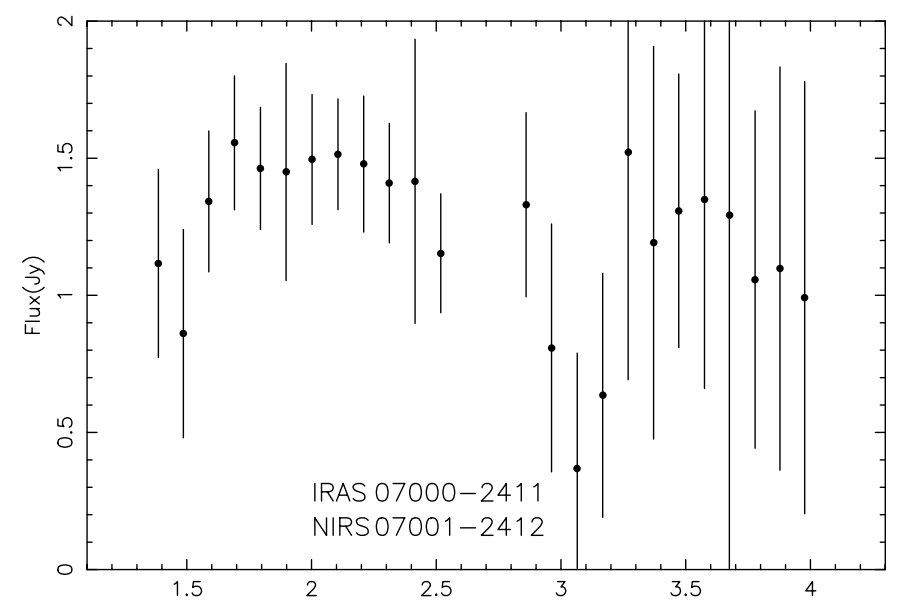

Fig. 9. Spectrum of NIRS 07001-2411 (CGCS 1517).

Acknowledgements. T. Le Bertre is grateful to the Institute of Space and Astronautical Science (ISAS) for several invitations to stay in Japan and for the permission to work on the IRTS data prior to its public release. This research has made use of the SIMBAD database, operated at CDS, Strasbourg, France. This work has been supported by the Paris Observatory through the fund BQR 2002. We are thankful to Dr. J. Kastner for several useful suggestions and for a careful reading of our manuscript.

\section{Appendix}

Groenewegen et al. (2002, G02) have evaluated distances and mass loss rates for a sample of 331 galactic carbon stars taken from the IRAS catalogue. They use data at 12 and $25 \mu \mathrm{m}$ to determine bolometric magnitudes, and $\mathrm{CO}$ rotational line spectra, to determine mass loss rates. They compare their results to those that we have obtained in Paper I for 11 sources which are in common. They find systematic discrepancies such that their distance estimates are larger by a factor 1.1 to 3.5 , and their mass loss rate estimates, by a factor $\sim 5$ (Table 8 , see also their Table 3). In Table 8 we added 3 sources in common between G02 and the present work.

Our estimates are based on NIRS data. We found one evidence of a misclassification and removed it from our comparison. (IRAS 17209-3318 is oxygen-rich: although its LRS index is 43 , its IRAS spectrum shows clearly a self-absorbed silicate feature mimicking an $11 \mu \mathrm{m}$ emission.) Our approach is statistical and we certainly do not expect a perfect agreement for every source. We use photometric data obtained at one phase, whereas the sources are known to be variable. Also, we assume an average luminosity of $8000 L_{\odot}$. We therefore might underestimate the distances to the most extreme carbon stars. Our treatment of the interstellar extinction is simplified, but this effect should be small because of the reduced extinction in the infrared ( $E_{\mathrm{K}} \leq 0.2$ for C-stars in our sample). The differences in $\dot{M}$ are of particular concern to us because we use a method that is in principle distance independent.

We note that, with the new processing of the NIRS data, our distance estimates stay close to those reported in Paper I. Also, two objects have an Hipparcos parallax: Y Tau and T Lyr. 
Table 6. Numbers of carbon-rich and oxygen-rich sources and their respective contributions to the replenishment of the ISM at different distances from the Galactic Plane.

\begin{tabular}{|c|c|c|c|c|c|c|c|c|}
\hline$d_{\mathrm{GC}}$ & $\begin{array}{r}\text { C-rich } \\
N\end{array}$ & $\sum \dot{M}_{-6}$ & $\begin{array}{r}\text { O-rich } \\
N\end{array}$ & $\sum \dot{M}_{-6}$ & $\begin{array}{r}\text { total } \\
N\end{array}$ & $\sum \dot{M}_{-6}$ & $\begin{array}{r}\text { ratio } \\
N\end{array}$ & $\begin{array}{r}\mathrm{C} /(\mathrm{O}+\mathrm{C}) \\
\sum \dot{M}_{-6}\end{array}$ \\
\hline $0-100 \mathrm{pc}$ & 42 & 16.89 & 187 & 91.24 & 229 & 108.13 & 0.18 & 0.16 \\
\hline $100-200 \mathrm{pc}$ & 34 & 23.38 & 79 & 40.25 & 113 & 63.63 & 0.30 & 0.37 \\
\hline $200-300 \mathrm{pc}$ & 18 & 16.10 & 64 & 41.79 & 82 & 57.88 & 0.22 & 0.28 \\
\hline $300-400 \mathrm{pc}$ & 11 & 14.67 & 47 & 16.41 & 58 & 31.09 & 0.19 & 0.47 \\
\hline $400-500 \mathrm{pc}$ & 4 & 1.78 & 35 & 7.68 & 39 & 9.47 & 0.10 & 0.19 \\
\hline $500-600 \mathrm{pc}$ & 6 & 3.52 & 21 & 17.79 & 27 & 21.31 & 0.22 & 0.17 \\
\hline $600-700 \mathrm{pc}$ & 1 & 0.03 & 29 & 12.92 & 30 & 12.95 & 0.03 & 0.00 \\
\hline $700-800 \mathrm{pc}$ & 4 & 0.25 & 22 & 20.96 & 26 & 21.20 & 0.15 & 0.01 \\
\hline $800-900$ pc & 2 & 0.21 & 16 & 16.83 & 18 & 17.04 & 0.11 & 0.01 \\
\hline $900-1000$ pc & 2 & 0.11 & 14 & 4.47 & 16 & 4.59 & 0.12 & 0.02 \\
\hline$>1000 \mathrm{pc}$ & 2 & 0.15 & 49 & 12.89 & 51 & 13.04 & 0.04 & 0.01 \\
\hline
\end{tabular}

Table 7. Numbers of carbon-rich and oxygen-rich sources and their respective contributions to the replenishment of the ISM for different mass-loss rates.

\begin{tabular}{crrrrrrrr}
\hline \hline$\dot{M}$ & C-rich & \multicolumn{3}{c}{ O-rich } & total & ratio & C/(O+C) \\
$\left(M_{\odot} \mathrm{yr}^{-1}\right)$ & $N$ & $\sum \dot{M}_{-6}$ & $N$ & $\sum \dot{M}_{-6}$ & $N$ & $\sum \dot{M}_{-6}$ & $N$ & $\sum \dot{M}_{-6}$ \\
\hline$\leq 10^{-7}$ & 74 & 4.19 & 302 & 12.55 & 376 & 16.74 & 0.20 & 0.25 \\
$10^{-7}-10^{-6}$ & 32 & 10.03 & 205 & 68.75 & 237 & 78.78 & 0.14 & 0.13 \\
$10^{-6}-10^{-5}$ & 19 & 52.69 & 52 & 142.25 & 71 & 194.95 & 0.27 & 0.27 \\
$10^{-5}-10^{-4}$ & 1 & 10.42 & 4 & 61.89 & 5 & 72.30 & 0.20 & 0.14 \\
\hline
\end{tabular}

Table 8. Comparison of distance and mass loss rate estimates obtained by Groenewegen et al. (2002, G02) and by us (Paper I and this work). The mass loss rates are in $10^{-6} M_{\odot} \mathrm{yr}^{-1}$, and the distances, in kpc.

\begin{tabular}{cccccccc}
\hline \hline IRAS name & $\begin{array}{c}\dot{M} \\
(\mathrm{G} 02)\end{array}$ & $\begin{array}{c}\dot{M} \\
\text { (Paper I) }\end{array}$ & $\begin{array}{c}\dot{M} \\
\text { (this work) }\end{array}$ & $\begin{array}{c}d \\
(\mathrm{G} 02)\end{array}$ & $\begin{array}{c}d \\
\text { (Paper I) }\end{array}$ & $\begin{array}{c}d \\
\text { (this work) }\end{array}$ & Comments \\
\hline $02596+6639$ & 6.43 & 1.58 & 1.92 & 4.88 & 2.53 & 2.49 & \\
$04449+4951$ & 8.86 & 3.04 & 3.71 & 2.07 & 1.94 & 1.80 & \\
$05426+2040$ & 1.17 & 0.04 & 0.05 & 1.25 & 0.40 & 0.42 & Y Tau, SRa, $\mathrm{d}_{\text {Hipp }}: 0.27 \mathrm{kpc}$ \\
$11318-7256$ & 5.75 & 0.92 & 1.09 & 0.72 & 0.60 & 0.63 & \\
$18306+3657$ & 3.46 & 0.05 & 0.08 & 1.38 & 0.38 & 0.39 & T Lyr, Lb, $\mathrm{d}_{\text {Hipp }: 0.63 \mathrm{kpc}}$ \\
$19248+0658$ & 4.90 & 1.15 & 2.35 & 2.02 & 1.37 & 1.35 & AFGL 2392, $\mathrm{d}_{\text {L97 }}: 2.0 \mathrm{kpc}$ \\
$20084-1425$ & 1.49 & 0.26 & - & 3.45 & 1.33 & - & R Cap, Mira \\
$21262+7000$ & 5.36 & 0.28 & 0.30 & 1.75 & 0.67 & 0.68 & AX Cep, Mira \\
$06230-0930$ & 6.35 & 3.36 & - & 2.19 & 4.46 & - & AFGL 935, $\mathrm{d}_{\text {L97 }}: 2.2 \mathrm{kpc}$ \\
$07098-2012$ & 14.1 & 4.44 & 4.33 & 1.66 & 1.18 & 1.24 & AFGL 1085, $\mathrm{d}_{\text {L97 }}: 1.8 \mathrm{kpc}$ \\
\hline $07217-1246$ & 4.83 & - & 4.30 & 1.86 & - & 1.32 & \\
$19029+2017$ & 9.18 & - & 4.47 & 2.60 & - & 1.61 & \\
$19108+1155$ & 12.9 & - & 1.16 & 3.05 & - & 1.90 & \\
\hline
\end{tabular}

The distances that we derive (Paper I and this work) are in better agreement with Hipparcos than those of G02. Three sources (IRAS 19248+0658, 06230-0930 and 07098-2012) are infrared sources which have been monitored and for which a distance has been determined through the period-luminosity relationship (Le Bertre 1997, L97). The distances found are 2.0, 2.2 and $1.8 \mathrm{kpc}$, resp., in better agreement with G02 than with Paper I and this work (but still within a factor 2). This is probably an effect of the variability which is large at $2 \mu \mathrm{m}$ for these sources (Le Bertre 1992).
On the other hand the mass loss rates of Le Bertre (1997) are in better agreement with Paper I and this work than with G02. This illustrates a fundamental difference between the 2 methods. G02 use a method based on the circumstellar CO emission which probes a region of typically $10^{17} \mathrm{~cm}$. Such a volume is filled in on a timescale of $\sim 10^{3}$ years or more. The determinations of G02 correspond to an average over such timescale. However, our method uses as an indicator of $\dot{M}$ the near-infrared emission that comes from a region of about $10^{15} \mathrm{~cm}$. We are therefore estimating $\dot{M}$ on a much 
Table 9. Comparison of mass loss rate estimates obtained by Groenewegen et al. (2002, G02), by us (t. w.), Loup et al. (1993, L93), Olofsson et al. (1993, O93) and Schöier \& Olofsson (2001, S01). The mass loss rates are in $10^{-6} M_{\odot} \mathrm{yr}^{-1}$, and the distances, in kpc.

\begin{tabular}{cccccccccccc}
\hline \hline IRAS name & $d$ & $\dot{M}$ & $d$ & $\dot{M}$ & $\dot{M}(\mathrm{G} 02)$ & $d$ & $\dot{M}$ & $d$ & $\dot{M}$ & $d$ & $\dot{M}$ \\
& $(\mathrm{G} 02)$ & $(\mathrm{G} 02)$ & $(\mathrm{t} . \mathrm{w})$. & $(\mathrm{t} . \mathrm{w})$. & d (t. w.) & $(\mathrm{L} 93)$ & $(\mathrm{L93})$ & $(\mathrm{O} 93)$ & $(\mathrm{O} 93)$ & $\begin{array}{c}d \\
(\mathrm{~S} 01)\end{array}$ & $(\mathrm{S} 01)$ \\
\hline $02596+6639$ & 4.88 & 6.43 & 2.49 & 1.92 & 1.67 & & & & & & \\
$04449+4951$ & 2.07 & 8.86 & 1.80 & 3.71 & 6.70 & & & & & & \\
$05426+2040$ & 1.25 & 1.17 & 0.42 & 0.05 & 0.13 & 0.57 & 0.85 & 0.50 & 0.45 & 0.31 & 0.40 \\
$11318-7256$ & 0.72 & 5.75 & 0.63 & 1.09 & 4.40 & 0.62 & 2.70 & & & & \\
$18306+3657$ & 1.38 & 3.46 & 0.39 & 0.08 & 0.28 & 1.20 & 2.60 & 0.51 & 0.07 & 0.34 & 0.07 \\
$19248+0658$ & 2.02 & 4.90 & 1.35 & 2.35 & 2.19 & & & & & & \\
$21262+7000$ & 1.75 & 5.36 & 0.68 & 0.30 & 0.81 & & & & & & \\
$07098-2012$ & 1.66 & 14.1 & 1.24 & 4.33 & 7.87 & 1.50 & 6.20 & & & \\
$07217-1246$ & 1.86 & 4.83 & 1.32 & 4.30 & 2.43 & 1.50 & 3.30 & & & \\
$19029+2017$ & 2.60 & 9.18 & 1.61 & 4.47 & 3.52 & & & & & \\
$19108+1155$ & 3.05 & 12.9 & 1.90 & 1.16 & 5.01 & & & & & \\
\hline
\end{tabular}

shorter timescale ( 20-50 years). As discussed in Paper I, we consider a present mass loss rate defined as an average over the last $\sim 20-50$ years, a lapse which is short compared to the stellar evolution of such sources. Indeed, setting aside the pulsation, and the possible effect of multiperiodicity (Fleischer et al. 1995), AGB outflows can be considered as stationary over such a timescale. On the other hand, we have growing evidence of variations over longer timescales (e.g. Mauron \& Huggins 2000). It is interesting to note that the 2 objects, for which the discrepancy is the largest $(>20)$, have optical counterparts which are not undergoing large amplitude pulsations (IRAS $05426+2040 \equiv$ Y Tau, SRa; IRAS $18306+3657 \equiv \mathrm{T}$ Lyr, $\mathrm{Lb}$; both with $\Delta V \sim 2$ ). It is difficult to believe that these objects are presently losing mass at rates of respectively 1.2 and $3.5 \times 10^{-6} M_{\odot} \mathrm{yr}^{-1}$ (G02). It is worth noting that important variations of mass loss rate, by a factor $\sim 10$ over the last $\sim 10^{3}$ years, have been reported for some stars, in general SR variables, which show composite $\mathrm{CO}$ rotational line profiles (Knapp et al. 1998). In some cases the mass loss variation from such stars may even be so large that one observes a "detached shell" (Olofsson et al. 1996). Therefore the comparison between the rates estimated by us and through the $\mathrm{CO}$ method are meaningful only for sources that did not undergo major changes during the last few $10^{3}$ years.

As noted by G02, a part of the discrepancy on $\dot{M}$ may be ascribed to the different distance estimates which are used, because with the CO method the $\dot{M}$ estimates depend on $d^{2}$. In Table 9 we correct the G02 $\dot{M}$ estimates by adopting our distance estimates (6th column). The new estimates are indeed in better agreement with our results. However, we should be careful in interpreting this "improvement", because our distance estimates may not be much better than those of G02, whereas our mass loss estimates are basically distance independent.

In the same table we collect results obtained by other authors who also derive mass loss rates from CO line observations: Loup et al. (1993, L93) and Olofsson et al. (1993) apply basically the same method as G02 to derive $\dot{M}$ estimates, whereas Schöier \& Olofsson (2001, S01) developed a detailed radiative transfer analysis. The L93 estimates approximately agree with those of G02. There is also a relative agreement between O93 and S01, and G02 for IRAS 05426+2040
(Y Tau), but surprisingly not for IRAS 18306+3657 (T Lyr). It seems that in the latter case, the $\mathrm{CO}$ results are discrepant (the expansion velocity quoted in the literature for this source varies between 12 and $26 \mathrm{~km} \mathrm{~s}^{-1}$ ) because the emission is weak. Clearly mass loss rates estimates derived from CO data depend also on the telescope beamwidth for nearby sources, on the modelling method and on several assumptions, such as the $\mathrm{CO}$ abundance or the $\mathrm{CO}$ density distribution. Recently, Schöier et al. (2002) improved the model of S01. They set an upper limit on the mass loss rate modulations to less than a factor of 5 over the past thousands of years. On the other hand, Kemper (2002), using CO rotational transitions from $J=2 \rightarrow 1$ up to $J=7 \rightarrow 6$, finds indications of mass loss variations on timescales similar to those observed by Mauron \& Huggins (2000).

These mass loss variations can explain why the $\mathrm{CO}$ method and the IR method give different results. However, one would expect to find also IR estimates larger than the $\mathrm{CO}$ estimates. These would correspond to sources that have recently begun losing mass at a very large rate. An object like IRAS 174113154, which is extremely red, $K-L^{\prime} \sim 7.9$ (Lépine et al. 1995), with a mass loss rate $\sim 2 \times 10^{-4} M_{\odot} \mathrm{yr}^{-1}$ (Jura \& Kleinmann 1989), yet very weak in CO (Kastner 1992), may be just such a case. This source is O-rich, and therefore does not appear in G02, but some carbon stars seem also to show the same effect. For instance, the mass loss rate estimate by the IR method for AFGL 3068 (Le Bertre 1997) is $\sim 3$ times larger than by the CO method (G02). Another possible example, OH/IR 26.5+0.6, has been studied by Justtanont et al. (1996) and Fong et al. (2002) who find evidence for a sudden increase of the mass loss rate, by a factor $\geq 50$, about 200 years ago. Other cases of deficiency of CO emission as compared to the IR energy distribution have been identified among OH/IR sources by Heske et al. (1990). However, we note that this deficiency could also be due to an extremely low gas temperature in the outer parts of dense circumstellar envelopes (possibly below $4 \mathrm{~K}$, as reported by Le Bertre \& Gérard (2001) in IRC +10216). Furthermore we caution that the evolutionary status of $\mathrm{OH} / \mathrm{IR}$ sources is not always clear, some being probably red supergiants rather than AGB stars. 
In summary, the 2 approaches are affected by various sources of uncertainty due to the methods and the assumptions made, and by the measurement errors. In addition, in the case of the $\mathrm{CO}$ method, the errors on the mass loss rate estimates may be amplified by the strong dependence on distance. Finally, when making comparisons it has to be kept in mind that the $\mathrm{CO}$ and IR mass loss rate estimates are not related to the same timescales.

\section{References}

Bouchet, P., Manfroid, J., \& Schmider, F. X. 1991, A\&AS, 91, 409

Cohen, M., Walker, R., Carter, B., et al. 1999, AJ, 117, 1864

Epchtein, N., Le Bertre, T., Lépine, J. R. D., et al. 1987, A\&AS, 71, 39

Fleischer, A. J., Gauger, A., \& Sedlmayr, E. 1995, A\&A, 297, 543

Fong, D., Justtanont, K., Meixner, M., \& Campbell, M. T. 2002, A\&A, 396, 581

Groenewegen, M. A. T., Sevenster, M., Spoon, H. W. W., \& Pérez, I. 2002, A\&A, 390, 511 (G02)

Guglielmo, F., Epchtein, N., Le Bertre, T., et al. 1993, A\&AS, 99, 31

Habing, H. J. 1998, A\&A, 200, 40

Heske, A., Forveille, T., Omont, A., van der Veen, W. E. C. J., \& Habing, H. J. 1990, A\&A, 239, 173

Ibata, R. A., Gilmore, G., \& Irwin, M. J. 1995, MNRAS, 277, 781

Jura, M., \& Kleinmann, S. G. 1989, ApJ, 341, 359

Jura, M., \& Kleinmann, S. G. 1990, ApJ, 364, 663

Justtanont, K., Skinner, C. J., Tielens, A. G. G. M., Meixner, M., \& Baas, F. 1996, ApJ, 456, 337

Kastner, J. H. 1992, ApJ, 401, 337

Kastner, J. H., Forveille, T., Zuckerman, B., \& Omont, A. 1993, A\&A, 275,163

Kemper, C. 2002, Thesis, University of Amsterdam

Knapp, G. R., Young, K., Lee, E., \& Jorissen, A. 1998, ApJS, 117, 209
Lattanzio, J., \& Forestini, M. 1999, IAU Symp., 191, 31

Le Bertre, T. 1992, A\&AS, 94, 377

Le Bertre, T. 1997, A\&A, 324, 1059 (L97)

Le Bertre, T., \& Gérard, E. 2001, A\&A, 378, L29

Le Bertre, T., Matsuura, M., Winters, J. M., et al. 2001, A\&A, 376, 997 (Paper I)

Le Bertre, T., and the RESPIRE consortium 2002, Proc. SF2A Scientific Highlights 2002, ed. F. Combes, \& D. Barret (EDP Sciences), 461

Le Bertre, T., \& Winters, J. M. 1998, A\&A, 334, 173

Lépine, J. R. D., Ortiz, R., \& Epchtein, N. 1995, A\&A, 299, 453

Loup, C., Forveille, T., Omont, A., \& Paul, J. F. 1993, A\&AS, 99, 291 (L93)

Matsuura, M., Yamamura, I., Murakami, H., Freund, M. M., \& Tanaka, M. 1999, A\&A, 348, 579

Mauron, N., \& Huggins, P. J. 2000, A\&A, 359, 707

Murakami, H., Freund, M. M., Ganga, K., et al. 1996, PASJ, 48, L41

Murakami, H., Noda, M., Cohen, et al. 2003, The calibration legacy of the ISO Mission, ed. L. Metcalfe, \& M. Kessler, ESA-SP 481, in press

Noda, M., Matsumoto, T., Murakami, H., et al. 1996, SPIE 2817, 248

Olofsson, H., Eriksson, K., Gustafsson, B., \& Carlström, U. 1993, ApJS, 87, 267 (O93)

Olofsson, H., Bergman, P., Eriksson, K., \& Gustafsson, B. 1996, A\&A, 311, 587

Pagel, B. E. J. 1997, Nucleosynthesis and Chemical Evolution of Galaxies (Cambridge University Press)

Schöier, F. L., \& Olofsson, H. 2001, A\&A, 368, 969 (S01)

Schöier, F. L., Ryde, N., \& Olofsson, H. 2002, A\&A, 391, 577

Stephenson, C. B. 1989, A General Catalogue of Cool Galactic Carbon Stars, 2nd ed. (Publications of the Warner and Swasey Observatory), $3, \mathrm{n}^{\circ} 2$

Yamamura, I., \& IRTS Team 1997, ASP Conf. Ser., 124, 72

Yamamura, I., Tanaka, M., Takahashi, H., et al. 2003, Exploiting the ISO Data Archive - Infrared Astronomy in the Internet Age, ed. C. Gry, S. Peschke, J. Matagne, et al., ESA-SP 511, 35 